\title{
Smith-Kingsmore syndrome: The first report of a Korean patient with the MTOR germline mutation c.5395G $>$ A p.(Glu1799Lys)
}

Dohwan Lee', Ja-Hyun Jang ${ }^{2}$, and Cha Gon Lee ${ }^{1, *}$

'Department of Pediatrics, Nowon Eulji Medical Center, Eulji University, Seoul, Korea

${ }^{2} \mathrm{GC}$ Genome, Yongin, Korea

Smith-Kingsmore syndrome (SKS; OMIM 616638), also known as macrocephaly-intellectual disability-neurodevelopmental disorder-small thorax syndrome (MINDS; ORPHA 457485), is a rare autosomal dominant disorder, the prevalence of which is not known. It is caused by a heterozygous germline mutation in MTOR (OMIM 601231). Ten different MTOR germline mutations in 27 individuals have been reported in the medical literature to date. These were all gain-of-function missense variants, and about half of the 27 individuals had c.5395G>A p.(Glu1799Lys) in MTOR. Here, I report for the first time a Korean patient with the heterozygous germline mutation c.5395G>A p.(Glu1799Lys) in MTOR. It was found to be a de novo mutation, which was identified by whole-exome sequencing and confirmed by Sanger sequencing. The patient showed typical clinical features of SKS, including macrocephaly/megalencephaly; moderate intellectual disability; seizures; behavioral problems; and facial dysmorphic features of curly hair, frontal bossing, midface hypoplasia, and hypertelorism.

Key words: MTOR, Germ-line mutation, Smith-Kingsmore syndrome, Megalencephaly, Intellectual disability.

\section{Introduction}

Smith-Kingsmore syndrome (SKS; OMIM 616638) was described as a rare autosomal dominant syndromic overgrowth and intellectual disability syndrome in 2013 by Smith et al. [1]. Also known as macrocephaly-intellectual disability-neurodevelopmental disorder-small thorax syndrome (MINDS; ORPHA 457485), it is caused by a heterozygous germline mutation in MTOR (OMIM 601231) on chromosome 1p36 [1]. SKS is characterized by intellectual disability; macrocephaly/hemicephaly/ megalencephaly; seizures; and facial dysmorphic features such as curly/wavy hair, frontal bossing, midface hypoplasia, small chin, and hypertelorism [2]. The prevalence of SKS remains unknown. Ten different MTOR germline mutations in 27 individuals from Europe, USA, and Australia (15 inherited in 7 families and 12 arising de novo) have been reported in the medical literature to date [1-8]. These were all gain-of-function missense variants. Among them, the MTOR mutation c.5395G>A p.(Glu1799Lys) has been found to occur most frequently (48\% [n=13/27]; 9 inherited in 4 families and 4 having arisen de novo) [2,5-8].

Here, We report for the first time a Korean girl who exhibited typical clinical features of SKS and harbored the MTOR germline mutation c.5395G>A p.(Glu1799Lys), identified by using nextgeneration sequencing.

\footnotetext{
Received: 26 November 2018, Revised: 31 December 2018, Accepted: 2 January 2019, Published: 30 June 2019

*Corresponding author: Cha Gon Lee, M.D. (iD https://orcid.org/0000-0001-7294-229X

Division of Child Neurology, Department of Pediatrics, Nowon Eulji Medical Center, Eulji University, 68 Hangeulbiseok-ro, Nowon-gu, Seoul 01830, Korea. Tel: +82-2-970-8222, Fax: +82-2-970-0068, E-mail: leechagon@eulji.ac.kr

Conflict of interest: The authors declare that they do not have any conflicts of interest.

(ac) This is an open-access article distributed under the terms of the Creative Commons Attribution Non-Commercial License (http://creativecommons.org/licenses/by-nc/4.0/) which permits unrestricted non-commercial use, distribution, and reproduction in any medium, provided the original work is properly cited.

(c) Copyright 2019 by the Korean Society of Medical Genetics and Genomics 


\section{Case}

An 18-year-old girl visited our clinic with unprovoked generalized tonic seizures. She was born prematurely at 36 weeks via vaginal delivery, with a birth weight of 2,600 g. Her mother and father were non-consanguineous individuals of Korean descent. The pedigree chart is illustrated in Fig. 1A. At birth, she showed tachypnea and was hospitalized in the neonatal intensive care unit (NICU). She had first been evaluated in the NICU for her facial dysmorphic features. The findings of all tests, including the neonatal metabolic test, hearing test, echocardiography, abdomen ultrasonography, brain ultrasonography, and G-band karyotyping, were normal at that time. She exhibited significant psychomotor developmental delay: she supported her head at 7 to 8 months, sat unassisted at 22 months, and walked independently at 15 to 16 months. In a physical examination at 18 years of age, she exhibited macrocephaly and obesity: height of 160.1 cm (-0.11 standard deviation [SD]), weight of $88.9 \mathrm{~kg}$ (+3.39 SD), body mass index of $34.7 \mathrm{~kg} / \mathrm{m}^{2}(+4.06 \mathrm{SD})$, and orbitofrontal cortex of $58.5 \mathrm{~cm}(+4.60 \mathrm{SD})$. She had distinctive facial features, including curly hair, frontal bossing, midface hypoplasia, hypertelorism with upward slanting palpebral fissures, a smooth philtrum, and a thin upper lip (Figs. 2A-C). She had calluses on the dorsal aspects of her fingers due to habitual hand-biting as a result of self-injurious behavior (Fig. 2D). Her neurodevelopmental evaluation was last checked at 16 years of age, and she demonstrated moderate intellectual disability, with a full-scale intelligence quotient of 41 on the Korean Wechsler Intelligence Scale for Children, fourth edition. Her social quotient of 33.75 and social age of 5.40 years also indicated moderate trainable intellectual disability according to the Social Maturity Scale. She demonstrated severe mental functional impairment, with a score of 15 according to the Children's Global Assessment Scale, and extremely severe mental functional impairment, with a score of 7 on the Clinical Global Impression Severity Scale. Chest radiography had not shown a small thorax at 5 years of age (Fig. 2E). Serial brain magnetic resonance imaging at 1,5 , and 7 years of age had shown scaphocephaly and megalencephaly (Figs. $2 \mathrm{~F}$ and $\mathrm{G}$ ). Electroencephalography at 18 years of age showed relatively low-amplitude background activity compared to that expected for her age; no epileptic-form discharges were seen.

The Institutional Review Board of the Nowon Eulji Medical Center (EMCS 2018-11-035) approved the use of human clinical materials and blood in this study. Written informed consent for the publication of medical photographs and genetic test results was obtained from the patient and her parents.

Cytogenetic evaluations, including rechecked G-band karyotyping at a resolution of 550 bands and 750K high-resolution genotyping SNP microarray (Affymetrix, Santa Clara, CA, USA) analysis, were conducted using the patient's peripheral blood lymphocytes, which yielded normal results. Genomic DNA was extracted from the peripheral blood leukocytes from her family members. Whole-exome sequencing (WES) was performed for the patient. SureSelect Human All Exon V5 (Agilent Technologies, Santa Clara, CA, USA) was used for library preparation, and sequencing was done on the Illumina NextSeq500 platform (Illumina Inc., San Diego, CA, USA), generating 2×150 bp pairedend reads at Green Cross Genome (Yongin, Korea). The heterozy-

B

A

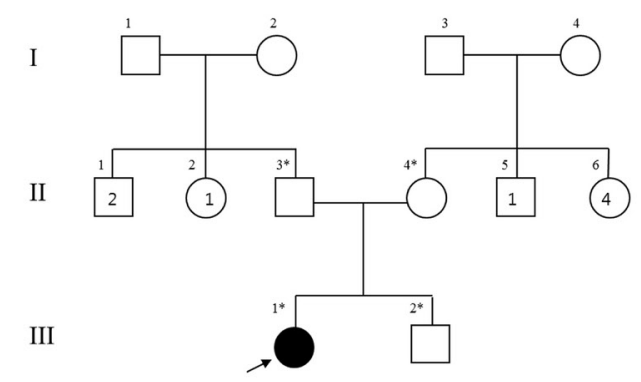

Mother (II-4)

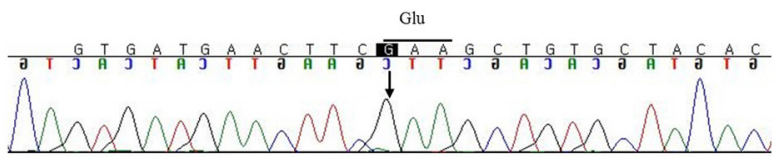

Father (II-3)

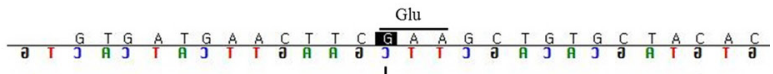

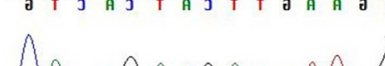

$\downarrow$

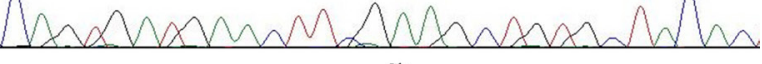

Brother (III-2)

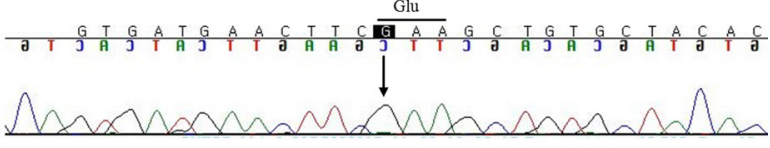

Patient (III-1)

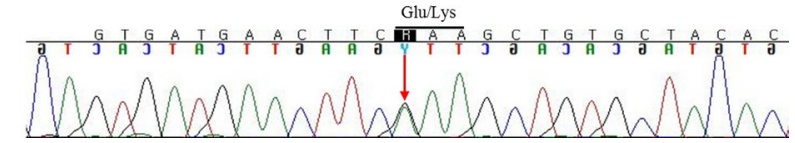

Fig. 1. (A) Pedigree of the patient's family. Asterisks indicate the sampled subjects. (B) Sanger sequencing confirmed the heterozygous MTOR variant c.5395G $>$ A p.(Glu1799Lys) in the patient (III-1) and the wild-type genotype in unaffected family members (II-3, II-4, and III-2). 

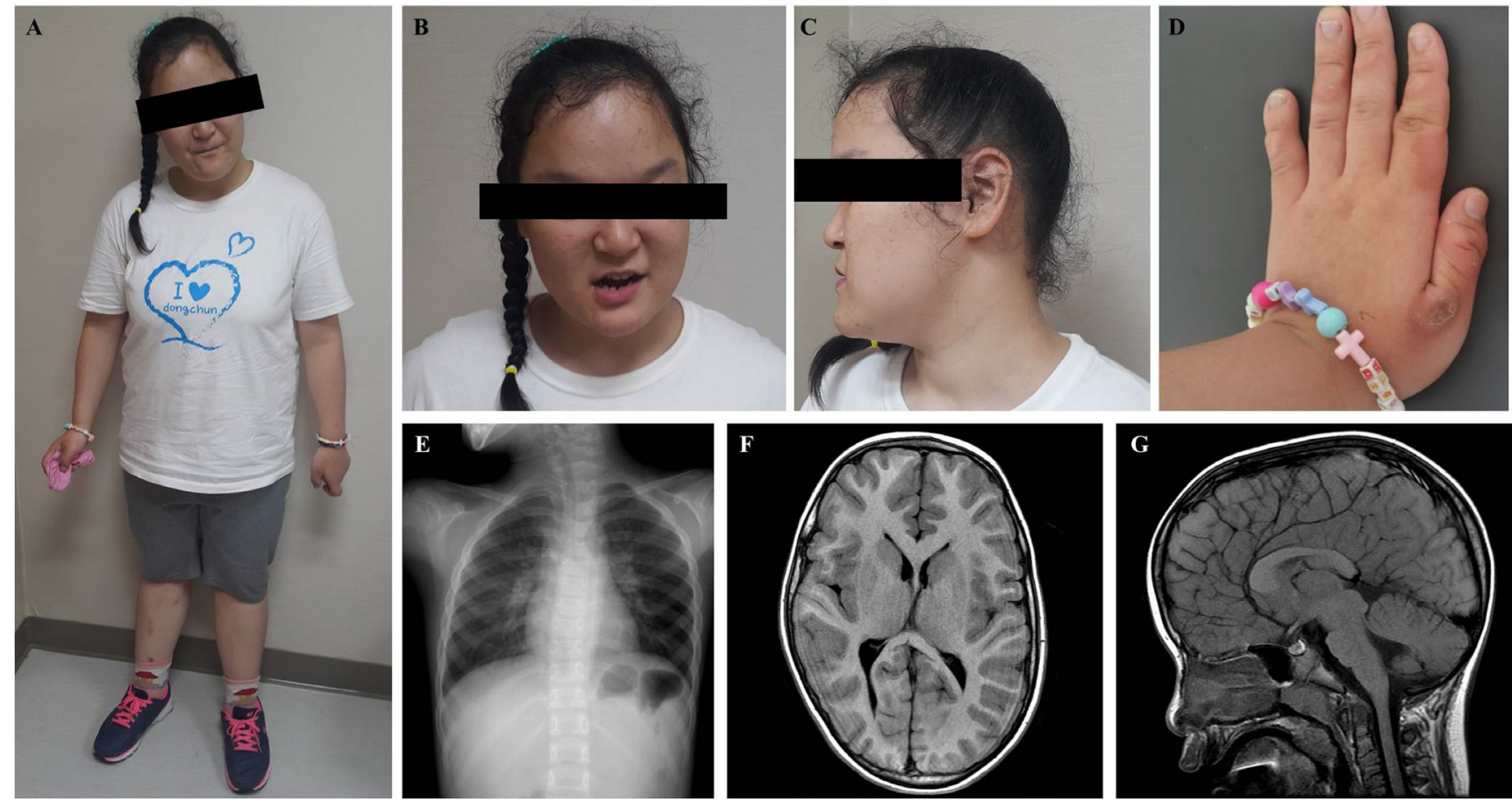

Fig. 2. (A-C) Photograph of the patient at 18 years of age. She showed curly hair, frontal bossing, midface hypoplasia, hypertelorism with upward slanting palpebral fissures, a smooth philtrum, and a thin upper lip. (D) Photograph of the left hand. There are multiple calluses on the dorsal aspects of the fingers due to habitual hand-biting owing to self-injurious behavior. (E) Chest X-ray shows no small thorax at 5 years of age. T1weighted axial $(F)$ and sagittal $(G)$ brain magnetic resonance imaging at the age of 7 years shows scaphocephaly and megalencephaly.

gous missense variant c.5395G>A p.(Glu1799Lys) of MTOR was identified on the basis of the reference sequence NM_004958.3 and was classified as a likely pathogenic variant with strong evidence of pathogenicity (PS1: same amino acid change as a previously established pathogenic variant, regardless of nucleotide change) and moderate evidence (PM2: absent from populations such as gnomAD and Korean Reference Genome Database) according to the guidelines of the American College of Medical Genetics and Genomics [9]. This was confirmed by Sanger sequencing in the patient and her parents. The results of Sanger sequencing are presented in Fig. 1B.

\section{Discussion}

MTOR is a highly conserved protein kinase, which is a master growth controller that nucleates two complexes: target of rapamycin complex 1 (TORC1) and TORC2 $[10,11]$. TORC1 is a key regulator of cell growth and proliferation and $m$ RNA translation, whereas TORC2 promotes actin cytoskeletal rearrangement, cell survival, and cell cycle progression $[10,11]$. MTOR is a component of the intracellular signaling pathway known as the phosphatidylinositol 3-kinase/Akt/mammalian target of rapamycin pathway, which has been actively studied for various therapeutic targets. Mutations of the genes encoding the components of the pathway are associated with several well-known syndromes such as tuberous sclerosis complex (OMIM 191100 and 613254) and PIK3CA-related overgrowth spectrum (OMIM 602501). A somatic mutation in MTOR was found to have caused focal cortical dysplasia type II (OMIM 607341), and a germline mutation in MTOR was reported to have caused SKS [2,3]. Although only a handful of SKS cases have been reported thus far (27 individuals with 10 different mutations), about half of them had the MTOR mutation c.5395G>A p.(Glu1799Lys). To date, there are no clear genotype-phenotype correlations [2]. The c.5395G >A variant is considered as a hotspot in SKS. MTOR contains several conserved functional domains [2]. The $\mathrm{N}$-terminus possesses 20 tandem HEAT (for Huntington, EF3, A subunit of PP2A, TOR 1) repeats, which serve as protein-protein interaction parts. The C-terminal domains contain a kinase domain, a catalytic domain containing an FKBP-rapamycin-binding domain, a FRAP-ATM-TTRAP (FAT) domain, and a FAT C-terminus domain [2]. The c.5395G>A p.(Glu1799Lys) variant affects the FAT domain of MTOR. Most variants of SKS patients cluster in the FAT [2]. This suggests that the mutations in the FAT domain may decrease the binding of the MTOR endogenous inhibitor Deptor (DEP-domain containing partner of TOR) and increase MTOR activity [2]. Thus, hotspot 
sequencing of MTOR could be considered as an initial genetic diagnostic test in patients with the typical clinical phenotype of SKS. However, numerous syndromes require many differential diagnoses to conclusively arrive at a diagnosis on the basis of clinical phenotypes alone. This is because of the limitations and complexity of genotype-phenotype correlations, as is the case for most monogenic disorders. Facial dysmorphic features of frontal bossing are similar to those of other syndromes such as Sotos syndrome (OMIM 117550). Macrocephaly and intellectual disability should be differentially diagnosed from several other overgrowth and intellectual disability syndromes including Tatton-Brown-Rahman syndrome (OMIM 615879) and Weaver syndrome (OMIM 277590) [12]. Thus, targeted multigene panel testing could be a more efficient diagnostic approach owing to its high coverage depth, as well as for appropriate differential diagnoses. However, unfortunately, this study was performed using WES.

Several SKS patients have been reported to have inherited the trait from parental germline mosaic mutations or gonadal mosaicism $[2,8]$. In the present study, Sanger sequencing was performed for the family members, but it had a low limit of detection for low-percentage germline mosaic variants. Moreover, in the present study, the test for detecting gonadal mosaicism in the patient' parents could not be performed. Despite these limitations, our patient was suspected to have a de novo mutation on the basis of Sanger sequencing of samples from the family members.

In summary, We report for the first time a Korean patient with the heterozygous germline mutation c.5395G>A p.(Glu1799Lys) in MTOR, suggesting its de novo origin, which was identified by WES and confirmed by Sanger sequencing.

\section{References}

1. Smith $L S$, Saunders $C$, Dinwiddie DL, Atherton AM, Miller N, Soden SE, et al. Exome sequencing reveals de novo germline mutation of the mammalian target of rapamycin (MTOR) in a patient with megalencephaly and intractable seizures. J Genome Exome 2013;2013:63-72.

2. Gordo G, Tenorio J, Arias P, Santos-Simarro F, García-Miñaur S, Moreno JC, et al. mTOR mutations in Smith-Kingsmore syndrome: four additional patients and a review. Clin Genet 2018;93:762-75.

3. Møller RS, Weckhuysen $S$, Chipaux M, Marsan E, Taly V, Bebin EM, et al. Germline and somatic mutations in the MTOR gene in focal cortical dysplasia and epilepsy. Neurol Genet 2016;2:e118.

4. Epi4K Consortium; Epilepsy Phenome/Genome Project; Allen AS, Berkovic SF, Cossette P, Delanty N, et al. De novo mutations in epileptic encephalopathies. Nature 2013;501:217-21.

5. Baynam G, Overkov A, Davis M, Mina K, Schofield L, Allcock R, et al. A germline MTOR mutation in Aboriginal Australian siblings with intellectual disability, dysmorphism, macrocephaly, and small thoraces. Am J Med Genet A 2015;167:1659-67.

6. Mroske C, Rasmussen K, Shinde DN, Huether R, Powis Z, Lu HM, et al. Germline activating MTOR mutation arising through gonadal mosaicism in two brothers with megalencephaly and neurodevelopmental abnormalities. BMC Med Genet 2015;16:102.

7. Mirzaa GM, Campbell CD, Solovieff N, Goold C, Jansen LA, Menon $S$, et al. Association of MTOR mutations with developmental brain disorders, including megalencephaly, focal cortical dysplasia, and pigmentary mosaicism. JAMA Neurol 2016;73:836-45.

8. Moosa $S$, Böhrer-Rabel H, Altmüller J, Beleggia $F$, Nürnberg $P$, Li Y, et al. Smith-Kingsmore syndrome: a third family with the MTOR mutation c.5395G>A p.(Glu1799Lys) and evidence for paternal gonadal mosaicism. Am J Med Genet A 2017;173:264-7.

9. Richards S, Aziz N, Bale S, Bick D, Das S, Gastier-Foster J, et al. Standards and guidelines for the interpretation of sequence variants: a joint consensus recommendation of the American College of Medical Genetics and Genomics and the Association for Molecular Pathology. Genet Med 2015;17:405-24.

10. Jacinto $E$, Loewith $R$, Schmidt $A$, Lin $S$, Rüegg MA, Hall $A$, et al. Mammalian TOR complex 2 controls the actin cytoskeleton and is rapamycin insensitive. Nat Cell Biol 2004;6:1122-8.

11. Hsu PP, Kang SA, Rameseder J, Zhang Y, Ottina KA, Lim D, et al. The mTOR-regulated phosphoproteome reveals a mechanism of mTORC1-mediated inhibition of growth factor signaling. Science 2011;332:1317-22.

12. Tatton-Brown K, Loveday C, Yost $S$, Clarke M, Ramsay E, Zachariou $A$, et al. Mutations in epigenetic regulation genes are a major cause of overgrowth with intellectual disability. Am J Hum Genet 2017; 100:725-36. 